PROCEEDINGS OF THE AMERICAN MATHEMATICAL SOCIETY

Volume 128, Number 1, Pages 245-253

S 0002-9939(99)05131-X

Article electronically published on May 11, 1999

\title{
A NOTE ON THE OSSERMAN CONJECTURE AND ISOTROPIC COVARIANT DERIVATIVE OF CURVATURE
}

\author{
NOVICA BLAŽIĆ, NEDA BOKAN, AND ZORAN RAKIĆ \\ (Communicated by Christopher Croke)
}

\begin{abstract}
Let $M$ be a Riemannian manifold with the Jacobi operator, which has constant eigenvalues, independent on the unit vector $X \in T_{p} M$ and the point $p \in M$. Osserman conjectured that these manifolds are flat or rankone locally symmetric spaces $(\nabla R=0)$. It is known that for a general pseudo-Riemannian manifold, the Osserman-type conjecture is not true and 4dimensional Kleinian Jordan-Osserman manifolds are curvature homogeneous. We show that the length of the first covariant derivative of the curvature tensor is isotropic, i.e. $\|\nabla R\|=0$. For known examples of 4-dimensional Osserman manifolds of signature $(--++)$ we check also that $\|\nabla R\|=0$. By the presentation of a class of examples we show that curvature homogeneity and $\|\nabla R\|=0$ do not imply local homogeneity; in contrast to the situation in the Riemannian geometry, where it is unknown if the Osserman condition implies local homogeneity.
\end{abstract}

\section{$\S 0$. INTRODUCTION}

Let $(M, g)$ be a 4-dimensional Kleinian (neutral) manifold, i.e. a pseudo-Riemannian manifold with a metric $g$ of signature $(--++)$. We denote its curvature tensor by $R$. The Jacobi operator $R_{X}: Y \mapsto R(Y, X) X$ is a symmetric endomorphism of $T_{p} M$ and $\mathcal{K}_{X}$ is its restriction to $X^{\perp}$ in $T_{p} M$.

For Riemannian manifolds, Osserman [16], based on joint results with Sarnak [17], has conjectured that if the eigenvalues of the Jacobi operator $\mathcal{K}_{X}$ are independent of the choice of unit vectors $X \in T_{p} M$ and of the choice $p \in M$, then either $M$ is locally a rank-one symmetric space or $M$ is flat. We have generalized in [3] the Osserman-type condition in the pseudo-Riemannian setup in terms of the Jordan form of $\mathcal{K}_{X}$, that is equivalent, especially for 4-dimensions, to the conditions in terms of the constancy of the minimal polynomial for $\mathcal{K}_{X}$. Namely, $M$ is spacelike (resp. timelike) Jordan-Osserman at $p$ if the Jordan form of $\mathcal{K}_{X}$ is independent of $X \in T_{p} M, g(X, X)=1$ (resp. $g(X, X)=-1$ ). If $M$ is spacelike (resp. timelike) Jordan-Osserman at every $p \in M$, one says $M$ is pointwise spacelike (resp. timelike) Jordan-Osserman. If the Jordan form of $\mathcal{K}_{X}$ is independent of $p \in M$, then $M$ is spacelike (resp. timelike) Jordan-Osserman.

Received by the editors November 6, 1997 and, in revised form, March 3, 1998

1991 Mathematics Subject Classification. Primary 53B30, 53C50.

Key words and phrases. Pseudo-Riemannian manifold, curvature tensor, Jacobi operator, Kleinian Osserman spacelike (timelike) manifold, Osserman conjecture, isotropicity.

Research partially supported by SFS, Project \#04M03. 
Many mathematicians have studied Osserman-type manifolds (see e.g. [9], [13], [14] for the Riemannian case; [1], [10] for Lorentzian spaces and [3] for 4-dimensional Kleinian manifolds). More details can be found in [2].

Jordan-Osserman manifolds with a metric of signature $(--++)$ are curvature homogeneous and their curvature invariants of the first and second order are constant. The main purpose of this paper is to study the third order curvature invariant $\|\nabla R\|^{2}$ for this class of manifolds. We show that the first covariant derivative of the curvature tensor is isotropic, i.e. $\|\nabla R\|=0$. Moreover, we check isotropicity of $\nabla R$ for all known examples of Osserman spaces in the general setting. We provide a class of examples of Jordan-Osserman manifolds which are not local homogeneous. Of course, in the general setting, Osserman spaces are not curvature homogeneous and consequently they are not locally homogeneous (cf. [12]).

This result, together with known results in the Riemannian and the Lorentzian cases, enable us to state the Osserman conjecture for timelike (spacelike) pseudoRiemannian manifolds related to the Riemannian Osserman conjecture, as follows:

Conjecture A. Every timelike or spacelike Jordan-Osserman manifold has the isotropic curvature derivative, i.e. $\|\nabla R\|=0$.

Since Conjecture A holds for all known examples of the Osserman spaces (see [7], [11]), we can state the stronger conjecture as follows:

Conjecture B. Every timelike or spacelike Osserman manifold has isotropic curvature derivative, i.e. $\|\nabla R\|=0$.

Remark. Also, an interesting question could be raised related to the isotropicity of higher curvature derivatives, $\left\|\nabla^{k} R\right\|=0$, for arbitrary $k$.

Curvature invariants are a very effective tool in Riemannian geometry and they build its relations with spectral geometry, topology and representation theory. So, F. Prüfer, F. Tricerri and L. Vanhecke have shown in [18] that if all curvature invariants are constant on the manifold, then this must be locally homogeneous. Anyhow, constancy of curvature invariants in the pseudo-Riemannian case is a necessary condition but not a sufficient one for local homogeneity of a manifold. In the Lorentzian case, examples are provided in [8] where the authors construct examples of 3- and 4-dimensional Lorentzian manifolds which are curvature homogeneous up to order one, but not locally homogeneous. The conformally flat plane metrics and the conditions to be locally homogeneous have been studied in [15].

Remark. In the Riemannian setting there exists an interesting conjecture, stated by L. Vanhecke, if the Osserman manifold has to be locally homogeneous (see [22]). For Lorentzian pointwise Osserman manifolds the corresponding conjecture holds, but Kleinian Jordan-Osserman manifolds may not be local homogeneous. This is clear from examples studied in $\S 4$.

\section{$\S 1$. Preliminaries}

Let us introduce some basic notations and notions. We denote by $e_{1}, \cdots, e_{4}$ a pseudo-orthonormal basis of $T_{p} M$. It means $g\left(e_{i}, e_{j}\right)=\varepsilon_{i} \delta_{i j}$, where $\varepsilon_{i}=-1$ for $i=1,2, \varepsilon_{i}=+1$ for $i=3,4$ and $j=1, \cdots, 4$. The corresponding dual 
forms of $e_{i}$ are $\theta^{i}$, i.e. $\theta^{i}\left(e_{j}\right)=\delta_{j}^{i}$. Let $\nabla$ be the Levi-Civita connection, and $R(X, Y): T_{p} M \longrightarrow T_{p} M$ its corresponding curvature operator given by

$$
R(X, Y) Z=\nabla_{X} \nabla_{Y} Z-\nabla_{Y} \nabla_{X} Z-\nabla_{[X, Y]} Z
$$

where $X, Y, Z, \cdots \in T_{p} M$. To omit rather complicated notations we denote vectors and vector fields by the same letters.

If $\omega_{i}^{j}$ and $\Omega_{i}^{j}$ are the connection 1-forms and the curvature 2-forms respectively, then we have

$$
\begin{aligned}
\nabla e_{i} & =\sum \omega_{i}^{s} e_{s}, \quad i=1, \cdots, 4, \\
\Omega_{i}^{j} & =\frac{1}{2} \sum R_{k l i}^{j} \theta^{k} \wedge \theta^{l},
\end{aligned}
$$

and the Cartan structural equations

$$
\begin{aligned}
d \theta^{i} & =-\sum \omega_{j}^{i} \wedge \theta^{j} \\
d \omega_{i}^{j} & =-\sum\left(\omega_{k}^{j} \wedge \omega_{i}^{k}+\Omega_{i}^{j}\right) \quad \text { (the second Bianchi identity). }
\end{aligned}
$$

Denote by $R_{j k l i}=R_{j k l}^{s} g_{s i}$. Let $R_{j k l i ; h}$ be the covariant derivatives of $R_{j k l i}$. One can see

$$
\begin{aligned}
\sum_{h} R_{j k l i ; h} \theta^{h} & =d\left(R_{j k l i}\right)-\sum_{s} R_{s k l i} \omega_{j}^{s}-\sum_{s} R_{j s l i} \omega_{k}^{s} \\
& -\sum_{s} R_{j k s i} \omega_{l}^{s}-\sum_{s} R_{j k l s} \omega_{i}^{s} .
\end{aligned}
$$

Studying the characteristic and minimal polynomials of $\mathcal{K}_{X}$, we have established in [3] four different types of spacelike (resp. timelike) 4-dimensional Kleinian JordanOsserman manifolds. We say $M$ is

(1) type Ia if $\mathcal{K}_{X}$ is diagonalizable;

(2) type Ib if the characteristic polynomial of $\mathcal{K}_{X}$ has a complex root;

(3) type II if the minimal polynomial of $\mathcal{K}_{X}$ has a double root $\alpha$;

(4) type III if the minimal polynomial of $\mathcal{K}_{X}$ has a triple root $\alpha$.

We deal with a null basis $\left\{f_{i}\right\}$ of $T_{p} M$ given by

$$
\begin{aligned}
& f_{1}=\frac{1}{\sqrt{2}}\left(e_{1}-e_{4}\right), \\
& f_{2}=\frac{1}{\sqrt{2}}\left(e_{2}+e_{3}\right), \\
& f_{3}=\frac{1}{\sqrt{2}}\left(e_{2}-e_{3}\right), \\
& f_{4}=\frac{1}{\sqrt{2}}\left(e_{1}+e_{4}\right),
\end{aligned}
$$

and its corresponding dual basis $\left\{\xi^{i}\right\}$ of $T_{p}^{*} M$. Denote by $\varphi_{i}^{j}$ connection 1-forms with respect to this basis. These 1 -forms $\varphi_{i}^{j}$ satisfy symmetry properties

$$
\begin{aligned}
& \varphi_{1}^{1}+\varphi_{4}^{4}=0, \varphi_{1}^{2}+\varphi_{3}^{4}=0, \varphi_{1}^{3}+\varphi_{2}^{4}=0, \varphi_{4}^{1}=\varphi_{1}^{4}=0, \\
& \varphi_{2}^{2}+\varphi_{3}^{3}=0, \varphi_{2}^{1}+\varphi_{4}^{3}=0, \varphi_{3}^{1}+\varphi_{4}^{2}=0, \varphi_{2}^{3}=\varphi_{3}^{2}=0
\end{aligned}
$$


(see [4], [6]). Nonzero components of the curvature tensor in the basis $\left\{f_{i}\right\}$ are respectively

$$
\begin{aligned}
& R_{1441}=R_{2332}=R_{1243}=R_{1342}=\alpha, \\
& R_{4334}=2,
\end{aligned}
$$

and

$$
\begin{aligned}
& R_{2143}=R_{3142}=R_{4141}=R_{2323}=\alpha, \\
& R_{3141}=R_{3123}=\sqrt{2},
\end{aligned}
$$

for types II and III Kleinian Jordan-Osserman spaces (see [3]).

\section{$\S 2$. The length of $\nabla R$ for Jordan-Osserman manifolds}

Geometrical properties of different types of spacelike (timelike) Jordan-Osserman manifolds may be very dissimilar (see [2], [3], [4], [6], [5], etc.). In this section we consider a common property of all 4-dimensional spacelike (timelike) JordanOsserman manifolds related to the isotropicity of $\nabla R$.

2.1 Theorem. Let $(M, g)$ be a 4-dimensional spacelike (timelike) Jordan-Osserman manifold. Then the length of $\nabla R$ vanishes, i.e. $\|\nabla R\|=0$.

Proof. The only nontrivial case is when the manifold $M$ is of type II or III. Indeed, it follows from [3] that a Jordan-Osserman type Ia 4-dimensional manifold is locally symmetric and type Ib does not exist.

So, let $M$ be a manifold of type II or III. Let $\left(g^{i j}\right)$ be the inverse matrix for the matrix of a metric $g=\left(g_{i j}\right)$. Then the lengths of the first covariant derivative of the curvature tensor is given by

$$
\|\nabla R\|^{2}=\sum g^{i x} g^{j y} g^{k z} g^{l t} g^{h u} R_{i j k l ; h} R_{x y z t ; u} .
$$

We now deal with the null basis $\left\{f_{i}\right\}$ and recall that nonzero components of the matrix of $g$ and inverse matrix $g^{-1}$ in this frame are as follows:

$$
g_{14}=g_{23}=g_{32}=g_{41}=g^{14}=g^{23}=g^{32}=g^{41}=-1 .
$$

We consider now type II Kleinian Jordan-Osserman manifolds. Hence we combine the relations (1.7) with (1.4) and (1.6) to compute nonzero 1-forms

$$
\begin{aligned}
& R_{3243 ; h} \xi^{h}=2 \varphi_{1}^{3}, \\
& R_{4143 ; h} \xi^{h}=2 \varphi_{1}^{3}, \\
& R_{4343 ; h} \xi^{h}=-4\left(\varphi_{1}^{1}+\varphi_{2}^{2}\right)=4 C .
\end{aligned}
$$

We have proved in [4, Theorem 2.3] that type II Kleinian Jordan-Osserman spaces yield

$$
\begin{aligned}
& \varphi_{1}^{3}\left(f_{1}\right)=\varphi_{1}^{3}\left(f_{2}\right)=0, \\
& \varphi_{1}^{3}\left(f_{3}\right)=2\left(\varphi_{1}^{1}+\varphi_{2}^{2}\right)\left(f_{1}\right), \\
& \varphi_{1}^{3}\left(f_{4}\right)=-2\left(\varphi_{1}^{1}+\varphi_{2}^{2}\right)\left(f_{2}\right) .
\end{aligned}
$$


We combine now (2.4) with (2.3) to find nonzero components $R_{i j k l ; h}$ of $\nabla R$

$$
\begin{aligned}
R_{4334 ; 1} & =R_{1434 ; 3}=R_{2334 ; 3}=-4 \tilde{C}_{1}, \\
-R_{4334 ; 2} & =R_{1434 ; 4}=R_{2334 ; 4}=4 \tilde{C}_{2}, \\
R_{4334 ; 3} & =-4 \tilde{C}_{3}, \\
R_{4334 ; 4} & =-4 \tilde{C}_{4},
\end{aligned}
$$

where $C=-\left(\varphi_{1}^{1}+\varphi_{2}^{2}\right)=\tilde{C}_{i} \xi^{i}$.

One can substitute (2.2) and (2.5) into (2.1) to check $\|\nabla R\|=0$.

We assume now $M$ is a type III Kleinian Jordan-Osserman manifold. Thus we use (1.4), (1.6) and (1.8) to obtain nonzero 1-forms

$$
\begin{array}{llrl}
R_{3131 ; h} \xi^{h} & =4 \sqrt{2} \varphi_{1}^{2}=-\sqrt{2} \hat{B}, & & R_{3132 ; h} \xi^{h}=\sqrt{2}\left(\varphi_{1}^{1}-\varphi_{2}^{2}\right)=-\sqrt{2} C, \\
R_{3141 ; h} \xi^{h} & =\sqrt{2}\left(-\varphi_{1}^{1}+\varphi_{2}^{2}\right)=\sqrt{2} C, & & R_{3142 ; h} \xi^{h}=-2 \sqrt{2} \varphi_{2}^{1}=\frac{1}{\sqrt{2}} \hat{A}, \\
R_{3232 ; h} \xi^{h} & =2 \sqrt{2} \varphi_{2}^{1}=-\frac{1}{\sqrt{2}} \hat{A}, & & R_{3241 ; h} \xi^{h}=-2 \sqrt{2} \varphi_{2}^{1}=\frac{1}{\sqrt{2}} \hat{A}, \\
R_{4141 ; h} \xi^{h}=2 \sqrt{2} \varphi_{2}^{1}=-\frac{1}{\sqrt{2}} \hat{A}, & &
\end{array}
$$

where 1-forms $\hat{A}, \hat{B}$ and $C$ are related with the connection forms $\varphi_{j}^{i}$ :

$$
\hat{A}=-4 \varphi_{2}^{1}, \quad \hat{B}=-4 \varphi_{1}^{2} \quad \text { and } \quad C=-\varphi_{1}^{1}+\varphi_{2}^{2} .
$$

Analogously to (2.4) (see [6, Theorem 2.3]), in the null frame $\left\{\xi^{i}\right\}$ these become

$$
\begin{aligned}
& \hat{A}=2 \tilde{C}_{2} \xi^{1}-2 \tilde{C}_{4} \xi^{3}, \\
& \hat{B}=\left(\sqrt{2} B_{1}+\tilde{C}_{3}\right) \xi^{1}+\tilde{C}_{1} \xi^{2}+\left(\sqrt{2} B_{2}-\tilde{C}_{1}\right) \xi^{i}-\tilde{C}_{3} \xi^{4}, \\
& C=\tilde{C}_{i} \xi^{i} .
\end{aligned}
$$

Similarly we find nonzero components of $\nabla R$ for the Kleinian spacelike (timelike) type III Jordan-Osserman manifolds

$$
\begin{aligned}
& R_{3131 ; 1}=-2 B_{1}-\sqrt{2} \tilde{C}_{3}, \quad R_{3131 ; 3}=-2 B_{2}+\sqrt{2} \tilde{C}_{1}, \\
& R_{3131 ; 2}=R_{3132 ; 1}=-R_{3141 ; 1}=-\sqrt{2} \tilde{C}_{1}, \\
& R_{3132 ; 2}=-R_{3141 ; 2}=R_{3232 ; 1}=R_{1414 ; 1}=-R_{3142 ; 1}=R_{3241 ; 1}=-\sqrt{2} \tilde{C}_{2}, \\
& R_{3132 ; 3}=-R_{3141 ; 3}=-R_{3131 ; 4}=-\sqrt{2} \tilde{C}_{3}, \\
& R_{3132 ; 4}=-R_{3141 ; 4}=R_{3142 ; 3}=-R_{3232 ; 3}=-R_{4141 ; 3}=R_{3241 ; 3}=-\sqrt{2} \tilde{C}_{4} .
\end{aligned}
$$

Then one can check that $\|\nabla R\|=0$ by straightforward computation.

Remark. One can see that the length of the curvature tensor of the 4-dimensional spacelike (timelike) Jordan-Osserman manifold of type II or III is $\|R\|^{2}=24 \alpha^{2}$.

Remark. All known examples of types II and III spacelike (timelike) Jordan-Osserman manifolds are nonflat but they are Ricci flat (this is for $\alpha=0$ ), and consequently all of them have the isotropic curvature tensor. 


\section{$\S 3 .\|\nabla R\|$ of some Kleinian Osserman spaces}

In this section, we will show that known examples of Kleinian Osserman manifolds have isotropic covariant derivative of the curvature tensor.

The known examples of Kleinian Osserman spaces have the metric tensor of one of the following types (for more details see [7], [11]):

(i)

$$
\begin{aligned}
g_{\left(f_{1}, f_{2}\right)}= & u_{3} f\left(u_{1}, u_{2}\right) d u_{1} \otimes d u_{1}+u_{4} f_{2}\left(u_{1}, u_{2}\right) d u_{2} \otimes d u_{2} \\
& +a\left[d u_{1} \otimes d u_{2}+d u_{2} \otimes d u_{1}\right] \\
& +b\left[d u_{1} \otimes d u_{3}+d u_{3} \otimes d u_{1}+d u_{2} \otimes d u_{4}+d u_{4} \otimes d u_{2}\right],
\end{aligned}
$$

where $\partial f_{1} / \partial u_{2}+\partial f_{2} / \partial / \partial u_{1}=0$.

(ii)

$$
g_{\phi}=\left[d u_{1} \otimes d u_{3}+d u_{3} \otimes d u_{1}+d u_{2} \otimes d u_{4}++d u_{4} \otimes d u_{2}\right]+\sum_{i, j=1}^{2} \phi_{i j} d u_{i} \otimes d u_{j},
$$

where $\phi_{i j}$ are functions in $u_{1}$ and $u_{2}$.

Then the characteristic polynomial of the Jacobi operator of the manifolds with metric of type (i) or (ii) is $p_{\lambda}\left(\mathcal{K}_{X}\right)=\lambda^{4}$, i.e., it is independent on the nonnull vector $X$, but the minimal polynomials, $m_{\lambda}\left(\mathcal{K}_{X}\right)$, can change degree from point to point (depending on the functions).

Now we can prove the following:

Proposition 3.1. Let $(M, g)$ be a 4-dimensional spacelike (timelike) Osserman manifold endowed with the metric of type (i) or (ii). Then the length of $\nabla R$ vanishes, i.e. $\|\nabla R\|=0$.

Proof. We give the proof for the Osserman manifolds of type (i). By direct computation one can find that the following components of the inverse matrix $g^{-1}$ vanish:

$$
g^{11}=g^{12}=g^{21}=g^{22}=g^{14}=g^{41}=0 .
$$

Using (1.4) one can find that all generic nonzero components of the first covariant derivative are:

$$
\begin{array}{llll}
R_{1221 ; 1}, & R_{1221 ; 2}, & R_{1221 ; 3}, & R_{1221 ; 4}, \\
R_{2131 ; 1}, & R_{2131 ; 2}, & R_{2142 ; 1}, & R_{2142 ; 2} .
\end{array}
$$

Now, one combines the formulas (3.1), (3.2) with (2.1) to find $\|\nabla R\|=0$. The proof for the metrics of type (ii) is similar, but simpler.

\section{§4. Local homogeneity of Kleinian Jordan-Osserman manifolds}

Let us recall some basic facts related to the local homogeneity. Let $\nabla^{i} R(p)$ denote the $i$ th covariant derivative of the Riemann curvature tensor at a point $p$. A pseudo-Riemannian manifold $(M, g)$ is said to be curvature homogeneous up to order $k \in \mathbb{N}[20]$, or to satisfy the condition $P(k)$, if for every two points $p, q \in M$, there exists a linear isometry $\varphi: T_{p} M \longrightarrow T_{q} M$ such that

$$
\varphi^{*}\left(\nabla^{i} R(q)\right)=\nabla^{i} R(p),
$$

for all $i=0, \cdots, k$. Singer showed that a Riemannian manifold $(M, g)$ is locally homogeneous if it satisfies the condition $P(k)$ for some $k>k_{M}$. For an $n$-dimensional 
manifold $M$ one can see $k_{M}<n(n-1) / 2$, where $k_{M}$ is the so-called Singer index of $(M, g)$. The equivalence theorem for $G$-structures due to Cartan and Sternberg [21] provides the generalization of these results to the pseudo-Riemannian case. For more details one can see [8].

Let us emphasize once again that Jordan-Osserman manifolds of signature $(--++)$ are Einstein curvature homogeneous up to order one with constant curvature invariants up to order 4 and $\|\nabla R\|=0$. Hence, these spaces are good candidates to be also locally homogeneous. The main purpose of this section is to construct a family of metrics with signature $(--++)$ which are type II JordanOsserman and not locally homogeneous. This shows that the Vanhecke type conjecture is not true (see the last remark in $\S 0$ and [22]). More precisely we prove:

Proposition 4.1. Let $(M, g)$ be a 4-dimensional manifold where $M \subseteq \mathbb{R}^{4}$ endowed with the metric

$$
\begin{aligned}
g= & a^{2} d u_{1} \otimes d u_{1}+b^{2} d u_{2} \otimes d u_{2} \\
& +d u_{1} \otimes d u_{3}+d u_{3} \otimes d u_{1}+d u_{2} \otimes d u_{4}+d u_{4} \otimes d u_{2}
\end{aligned}
$$

with respect to the standard Descartes coordinate system $\left(u_{1}, \cdots, u_{4}\right)$, where $a=$ $a\left(u_{1}, u_{2}\right), b=b\left(u_{1}, u_{2}\right)$ and $a, b \in C^{\infty}(M)$. Then $(M, g)$ is:

(i) a type II Jordan-Osserman manifold if $\frac{\partial^{2} a^{2}}{\partial u_{2}^{2}}+\frac{\partial^{2} b^{2}}{\partial u_{1}^{2}}>0$ everywhere on $M$;

(ii) curvature homogeneous for arbitrary $a, b \in C^{\infty}(M)$;

(iii) not locally homogeneous for $a, b$ satisfying $\delta=\delta\left(u_{1}, u_{2}\right) \neq$ const, where

$$
\delta\left(u_{1}, u_{2}\right)=\frac{64}{\left(\frac{\partial^{2} a^{2}}{\partial u_{2}^{2}}+\frac{\partial^{2} b^{2}}{\partial u_{1}^{2}}\right)^{5}}\left(\frac{\partial^{3} a^{2}}{\partial u_{2}^{3}}+\frac{\partial^{3} b^{2}}{\partial u_{1}^{2} \partial u_{2}}\right)^{2}\left(\frac{\partial^{3} a^{2}}{\partial u_{2}^{2} \partial u_{1}}+\frac{\partial^{3} b^{2}}{\partial u_{1}^{3}}\right)^{2} .
$$

Proof. We denote $E_{i}=\partial / \partial u_{i}(i=1, \cdots, 4)$. One can check that the vector fields

$$
\begin{aligned}
& F_{1}=-\frac{a}{\sqrt{2}} E_{3}, \\
& F_{2}=-\beta b E_{4}, \\
& F_{3}=\frac{1}{b \beta} E_{2}-\frac{b}{2 \beta} E_{4}, \\
& F_{4}=\frac{\sqrt{2}}{a} E_{1}-\frac{a}{\sqrt{2}} E_{3},
\end{aligned}
$$

form a null frame field for $g$; i.e.

$$
g\left(F_{1}, F_{4}\right)=g\left(F_{2}, F_{3}\right)=-1
$$

are the only nonvanishing components of the metric tensor $g$, where $\beta \in C_{+}^{\infty}(M)$. If we choose

$$
\beta^{2}=\frac{1}{2 a^{2} b^{2}}\left(\frac{\partial^{2} a^{2}}{\partial u_{2}^{2}}+\frac{\partial^{2} b^{2}}{\partial u_{1}^{2}}\right),
$$

then nonzero components of the curvature tensor $R$ and its first covariant derivative $\nabla R$ are given with respect to the null frame field $F_{i}$ by

$$
\begin{aligned}
R_{3434} & =-2, \\
R_{3434 ; 3} & =\frac{-1}{(b \beta)^{3} a^{2}}\left(\frac{\partial^{3} a^{2}}{\partial u_{2}^{3}}+\frac{\partial^{3} b^{2}}{\partial u_{1}^{2} \partial u_{2}}\right), \\
R_{3434 ; 4} & =-\frac{\sqrt{2}}{a^{3}(b \beta)^{2}}\left(\frac{\partial^{3} a^{2}}{\partial u_{2}^{2} \partial u_{1}}+\frac{\partial^{3} b^{2}}{\partial u_{1}^{3}}\right) .
\end{aligned}
$$

This means $P(0)$ is fullfield, i.e. $(M, g)$ is curvature homogeneous and hence (ii) is satisfied. We now use $R\left(F_{4}, F_{3}\right) F_{3}=-2 F_{1}, R\left(F_{4}, F_{3}\right) F_{4}=-2 F_{2}$ and 
$R\left(F_{4}, F_{3}\right) F_{i}=0(i=1,2)$ to see that $F_{1}$ and $F_{2}$ are eigenvectors of the symmetric endomorphism $R\left(F_{4}, F_{3}\right)$, corresponding to the double eigenvalue $\lambda=0$. By straightforward computations one can check (i) (see also [5] and [19]). Moreover for $\frac{\partial^{2} a^{2}}{\partial u_{2}^{2}}+\frac{\partial^{2} b^{2}}{\partial u_{1}^{2}}=0$ it follows that $(M, g)$ is flat.

Assume now that $M$ is locally homogeneous. It means that for each two points $p, q \in M$, there exists a linear isometry $\varphi: T_{p} M \longrightarrow T_{q} M$ such that

$$
\varphi^{*} R(q)=R(p), \quad \varphi^{*}(\nabla R(q))=\nabla R(p) .
$$

There exists also a null frame $V_{1}, \cdots, V_{4}$ around $p \in M$, obtained by the local isometries. The components $R_{i j k l}, R_{i j k l ; m}$ are constant along the manifold $M$. It is shown that

$$
\begin{aligned}
& V_{1}=d^{2} f F_{1}, \\
& V_{2}=d f^{2} F_{2}, \\
& V_{3}=c F_{1}+d F_{3}, \\
& V_{4}=e F_{2}+f F_{4},
\end{aligned}
$$

where $c, d, e, f$ are smooth functions in a neighborhood of $p \in M$ such that $(d f)^{2}=1$ and $c f+d e=0$. But, by straightforward computations, using (4.7) and (4.9) one can see $\left(R_{3434 ; 3} \cdot R_{3434 ; 4}\right)^{2}=\delta\left(u_{1}, u_{2}\right)$. Consequently, for $\delta \neq$ const, $M$ is not locally homogeneous.

Remark. The curvature tensor for this metric is recurrent for arbitrary functions $a\left(u_{1}, u_{2}\right), b\left(u_{1}, u_{2}\right) \in C^{\infty}(M)$ (see [19] and [5]).

Remark. One can check that $a=b=u_{1} u_{2}$ satisfy all conditions in Proposition 4.1 on $M=\mathbb{R}^{4} \backslash\{0\}$ since $\delta\left(u_{1}, u_{2}\right)=C \frac{u_{1}^{2} u_{2}^{2}}{\left(u_{1}^{2}+u_{2}^{2}\right)^{5}}, C=$ const.

\section{ACKNOWLEDGMENT}

We thank the referee for suggestions and comments to improve our paper and L. Vanhecke for pointing out the problem of relations between Osserman and local homogeneity conditions. We are also grateful to M. Djorić for several useful discussions.

\section{REFERENCES}

[1] N. Blažić, N. Bokan and P. Gilkey, A Note on Osserman Lorentzian manifolds, Bull. London Math. Soc. 29 (1997), 227-230. MR 97m:53111

[2] N. Blažić, N. Bokan, P. Gilkey and Z. Rakić, Pseudo-Riemannian Osserman manifolds, (preprint 1997).

[3] N. Blažić, N. Bokan and Z. Rakić, Characterization of 4-dimensional Osserman pseudo-Riemannian manifolds, (preprints 1995, 1997).

[4] - Characterization of type II Osserman manifolds in terms of connection forms, (preprint 1997).

[5] - Recurrent spacelike (timelike) Osserman spaces, to appear, Bull. Acad. Serbe Sci. Arts Cl. Sci Math. Natur.

[6] The first order PDE system for type III Osserman manifolds, Publ. de l'Inst. Math. 62(76) (1997), 113-119. MR 98m:53029

[7] A. Bonome, R. Castro, E. Garcia-Rio, L. M. Hervella, R. Vázquez-Lorenzo, Nonsymmetric Osserman indefinite Kähler manifolds, Proc. Amer. Math. Soc. 126 (1998), 2763-2769. MR 98m:53088

[8] P. Bueken, L. Vanhecke, Examples of curvature homogeneous Lorentz metrics, Class. Quantum Grav. 14 (1997), L93-L96. MR 98c:53073 
[9] Q. S. Chi, A curvature characterization of certain locally rank-one symmetric spaces, J. Diff. Geom. 28 (1988), 187-202. MR 90a:53060

[10] E. García-Rio, D. N. Kupeli and M. E. Vázquez-Abal, On a problem of Osserman in Lorentzian geometry, Differential Geometry and its Applications 7 (1997), 85-100. MR 98a:53099

[11] E. García-Rio, M. E. Vázquez-Abal and R. Vázquez-Lorenzo, Nonsymmetric Osserman pseudo Riemannian manifolds, Proc. Amer. Math. Soc. 126 (1998), 2771-2778. MR 98m:53089

[12] E. García-Rio, D. N. Kupeli, M. E. Vázquez-Abal, R. Vázquez-Lorenzo, Osserman affine connections and their Riemann extensions, preprint, 1997.

[13] P. Gilkey, Manifolds whose curvature operator has constant eigenvalues at the basepoint, J. Geom. Anal. 4 (1994), 155-158. MR 95f:53084

[14] P. Gilkey, A. Swann and L. Vanhecke, Isoparametric geodesic spheres and a conjecture of Osserman concerning the Jacobi operator, Quart. J. Math. Oxford. 46 (1995), 299-320. MR 96h:53051

[15] A. Koutras, C. Mcintosh, A metric with no invariants, Class. Quantum Grav. 13 (1996), L47-9. MR 97g:83027

[16] R. Osserman, Curvature in the eighties, Amer. Math. Monthly 97 (1990), 731-756. MR 91i:53001

[17] R. Osserman and P. Sarnak, A new curvature invariant and entropy of geodesic flows, Invent. Math. 77 (1984), 455-462. MR 86a:58054

[18] F. Prüfer, F. Tricerri, L. Vanhecke, Curvature invariants, differential operators and local homogeneity, Trans. Am. Math. Soc. 348 (1996), 4643-4652. MR 97a:53074

[19] H. S. Ruse, A. G. Walker, T. J. Willmore, Harmonic spaces, Cremonese, Rome, 1961. MR 25:5456

[20] I. M. Singer, Infinitesimally homogeneous spaces, Commun. Pure Appl. Math. 13 (1960), 685-697. MR 24:A1100

[21] S. Sternberg, Lectures on Differential Geometry,, Prentice-Hall, Englewood Cliffs, NJ, 1964. MR 33:1797

[22] L. Vanhecke, Scalar curvature invariants and local homogeneity, Rend. Circ. Mat. Palermo 49 suppl. (1997), 275-287. CMP 98:09

Faculty of Mathematics, University of Belgrade, Studentski trg 16, P.P. 550, 11000 Belgrade, Yugoslavia

E-mail address: blazicn@matf.bg.ac.yu

E-mail address: neda@matf.bg.ac.yu,

E-mail address: zrakic@matf.bg.ac.yu 